Marquette University

e-Publications@Marquette

School of Dentistry Faculty Research and

Publications

Dentistry, School of

$1-2015$

\title{
Immunohistochemical Expression of Matrix Metalloprotease-2 and Matrix Metalloprotease-9 in the Disks of Patients with Temporomandibular Joint Dysfunction
}

\author{
Luis Eduardo Almeida \\ Marquette University, luis.almeida@marquette.edu \\ Karina Caporal \\ Pontificia Universidade Catolica do Parana \\ Viviane Ambros \\ Pontificia Universidade Catolica do Parana \\ Marina Azevedo \\ Pontificia Universidade Catolica do Parana \\ Lucia Noronha \\ Pontificia Universidade Catolica do Parana
}

See next page for additional authors

Follow this and additional works at: https://epublications.marquette.edu/dentistry_fac

Part of the Dentistry Commons

\section{Recommended Citation}

Almeida, Luis Eduardo; Caporal, Karina; Ambros, Viviane; Azevedo, Marina; Noronha, Lucia; Leonardi, Rosy; and Trevilatto, Paula C., "Immunohistochemical Expression of Matrix Metalloprotease-2 and Matrix Metalloprotease-9 in the Disks of Patients with Temporomandibular Joint Dysfunction" (2015). School of Dentistry Faculty Research and Publications. 52.

https://epublications.marquette.edu/dentistry_fac/52 


\section{Authors}

Luis Eduardo Almeida, Karina Caporal, Viviane Ambros, Marina Azevedo, Lucia Noronha, Rosy Leonardi, and Paula C. Trevilatto 


\title{
Immunohistochemical Expression of Matrix Metalloprotease-2 And Matrix Metalloprotease-9 In the Disks of Patients with Temporomandibular Joint Dysfunction
}

\author{
Luis Eduardo Almeida
}

School of Dentistry, Department of Oral Sciences, Oral Surgery,

Marquette University,

Milwaukee, WI

Karina Caporal

Pontificia Universidade Catolica do Parana,

Curitiba, Brazil

Viviane Ambros

Pontificia Universidade Catolica do Parana,

Curitiba, Brazil

Marina Azevedo

Pontificia Universidade Catolica do Parana,

Curitiba, Brazil 
NOT THE PUBLISHED VERSION; this is the author's final, peer-reviewed manuscript. The published version may be accessed by following the link in the citation at the bottom of the page.

\author{
Lucia Noronha \\ Pontificia Universidade Catolica do Parana, \\ Curitiba, Brazil \\ Rosy Leonardi \\ Department of Surgical Sciences, University of Catania, \\ Catania, Italy \\ Paula C. Trevilatto \\ Pontificia Universidade Catolica do Parana, \\ Curitiba, Brazil
}

\begin{abstract}
Purpose: Matrix metalloproteases (MMPs) are tissue-remodeling enzymes that function during the remodeling process, such as in immune-inflammatory diseases. Metalloprotease-2 (MMP-2) and metalloprotease-9 (MMP-9) are gelatinases that degrade several types of extracellular matrix collagen. It is hypothesized that in temporomandibular joint (TMJ) dysfunction, MMP-2 and MMP-9 expression levels may be elevated. Therefore, the objective of this study is to determine the association of MMP-2 and MMP-9 expression with temporomandibular joint dysfunction using an immunohistochemical approach to evaluate the joint disk.
\end{abstract}

Material and Methods: A total of 45 human temporomandibular joint samples were collected, with 36 samples in the test group (patients with anterior disk displacement with reduction $(n=29)$ and without reduction $(n=7))$ and nine samples in the control group. The immunostaining of the TMJ disks was statistically compared between the groups $(P<0.05)$.

Results: There was a statistically significant difference for the area of MMP-2 immunostaining between the control group and the displacement disks with reduction group (ADDwR) $(P=0.048)$ and between the groups with disk displacement and without reduction (ADDwoR) $(P=0.029)$. The expression of MMP-2 was significantly elevated in the ADDwoR group.

Conclusion: No statistically significant difference was found between the variable area of MMP-9 expression in the disk with and without disk displacement, as determined by immunohistochemical analysis. However, there was an elevation of MMP-2 expression in the disks of patients with displacement and without reduction (more severe alteration).

Journal of Oral Pathology \& Medicine, Vol 44, No. 1 (January 2015): pg. 75-79. DOI. This article is (c) Wiley and permission has been granted for this version to appear in e-Publications@Marquette. Wiley does not grant permission for this article to be further copied/distributed or hosted elsewhere without the express permission from Wiley. 
NOT THE PUBLISHED VERSION; this is the author's final, peer-reviewed manuscript. The published version may be accessed by following the link in the citation at the bottom of the page.

\section{Introduction}

The temporomandibular joint (TMJ) is a bilateral joint that adapts and remodels according to functional demands. The fibrous cartilaginous disk in the TMJ consists of regularly arranged collagen fibers and fibroblast-like cells. It plays an important role in distributing the pressure loads of articular surfaces of the condyle and glenoid fossa during joint movements. $\frac{1}{}$ When the disk is overloaded and exceeds its remodeling capability, it may suffer structural changes, such as the fragmentation of compact collagen fibers or an alteration of synovial cells, which cover the disk and are responsible for its lubrication and can lead to TMJ disorders. $\underline{\underline{2}}$ The pathogenesis of TMJ dysfunction is multifactorial, but the early identification of the major contributing factor is critical for improved treatment outcomes. Of the TMJ dysfunctions that may lead to disk degeneration, anterior disc displacement is generally observed in TMJ patients ${ }^{3}$ and often precedes osseous remodeling, disk perforation, and osteoarthritic changes. 4

The inflammatory process is a coordinated response against trauma and infection. However, when the initiating inflammatory agent is not removed, this physiological response may lead to pathological conditions. Matrix metalloproteinases (MMPs), a family of 20 proteolytic enzymes that degrade all types of collagen, proteoglycans, and other components of extracellular matrix are among the enzymes found in the physiological and inflammatory tissue environments. ${ }^{5}$ Their activity is essential for remodeling and adapting cartilage and other tissues. $\underline{6}$ The tissue inhibitors of metalloproteinases (TIMPs) work together with the MMPs during physiological alterations, $\underline{\underline{ }}$ but under inflammatory conditions, the excess activity and overexpression of MMPs results in tissue destruction. The MMPs are divided into the following subgroups: collagenases (MMP-1, MMP-8, and MMP-13) that degrade fibrillar collagen types I, II, and III; stromelysins (MMP-3 and MMP-11) that degrade proteoglycans and non-helical regions of collagens; and gelatinases (MMP-2 and MMP-9), which degrade the specific collagen types I, IV, V, and XI. ${ }^{8}$

MMP-2 and MMP-9 are produced primarily by inflammatory cells, and their expression is regulated at the transcriptional level by inflammatory mediators, such as interleukin-1. 9 MMP-2 and MMP-9 are

Journal of Oral Pathology \& Medicine, Vol 44, No. 1 (January 2015): pg. 75-79. DOI. This article is @ Wiley and permission has been granted for this version to appear in e-Publications@Marquette. Wiley does not grant permission for this article to be further copied/distributed or hosted elsewhere without the express permission from Wiley. 
synthesized as latent proenzymes, which are cleaved and activated outside the cells. MMP-2 and MMP-9 are increased in the serum and synovial fluids of arthritic patients and are considered markers of arthritis and are also associated with cartilage degradation in animal experiments. 10 MMP-2 was detected in early-stage osteoarthritis patients, whereas MMP-9 was highly expressed in patients with anterior disk displacement without reduction and advanced osteoarthritis. $\frac{11}{1}$ However, the sample was collected from synovial fluid, and the state of disk degradation was unknown during at that time. To better understand the progressive stage of the disease, it is important to acquire a detailed clinical evaluation and knowledge of the TMJ degenerating mechanisms. $\underline{12}$

Although the involvement of several MMPs in TMJ dysfunction is known, little has been reported regarding which MMP enzymes are involved in the TMJ disk disarrangements, and the elucidation of this relationship may contribute to improved diagnosis and treatment of TMJ patients. The majority of studies have been performed with synovial fluid, $\underline{13}$ animal models, $\underline{\underline{13}, 14}$ or cadavers. $\underline{15}$ However, MMP-2 and MMP-9 expressions have not been studied in the degenerated disks of human TMJ patients. Therefore, the objective of this study is to determine the association of MMP-2 and MMP-9 expressions in the disks of patients with temporomandibular joint dysfunction using an immunohistochemical approach to evaluate the joint disk.

\section{Materials and methods}

\section{Sample selection}

The sample set comprised 45 disk specimens (Table 1 ) from 33 patients, male and female, with a mean age of 32.36 years (17-57). A total of $36 \mathrm{TMJ}$ displaced disk specimens were collected from patients treated between 2002 and 2009 at the Evangelic University Hospital of Curitiba, Brazil. Nine virtually unaffected human TMJ disks were evaluated with the pathological material. The study was approved by the Ethical Committee on Research at Pontifical Catholic University of Paraná, Brazil, according to Resolution 196/96 of the National Health Council and approved under registration number 104.

Journal of Oral Pathology \& Medicine, Vol 44, No. 1 (January 2015): pg. 75-79. DOI. This article is (c) Wiley and permission has been granted for this version to appear in e-Publications@Marquette. Wiley does not grant permission for this article to be further copied/distributed or hosted elsewhere without the express permission from Wiley. 
Table 1. Baseline clinical characteristics of the study group with and without TMJ dysfunction

\section{Patient Race Sex Age Diagnoses Affected Affected side $(n=33) \quad$ (years) side (Right) (Left)}

1. ADDwoR, anterior disk displacement without reduction; ADDwR, anterior disk displacement with reduction; C FX, condylar fracture; $\mathrm{CH}$, condylar hyperplasia.

\begin{tabular}{|c|c|c|c|c|c|}
\hline 1 & Caucasian & Female & 35 & DDwoR & $X$ \\
\hline 2 & Caucasian & Female & 24 & DDwoR & \\
\hline 3 & Caucasian & Female & 23 & DDwoR & $x$ \\
\hline 4 & Caucasian & Female & 45 & DDwoR & $x$ \\
\hline 5 & Caucasian & Female & 32 & DDwoR & $x$ \\
\hline 6 & Caucasian & Female & 30 & ADDwR & \\
\hline 7 & $\begin{array}{l}\text { Afro- } \\
\text { American }\end{array}$ & Female & 20 & ADDwR & \\
\hline 8 & $\begin{array}{l}\text { Afro- } \\
\text { American }\end{array}$ & Female & 57 & ADDwR & \\
\hline 9 & Caucasian & Female & 33 & ADDwR & $x$ \\
\hline 10 & Caucasian & Female & 25 & ADDwR & $x$ \\
\hline 11 & Caucasian & Female & 22 & ADDwR & $x$ \\
\hline 12 & Caucasian & Female & 42 & ADDwR & $x$ \\
\hline 13 & Caucasian & Female & 56 & ADDwR & $x$ \\
\hline 14 & Caucasian & Female & 26 & ADDwR & $x$ \\
\hline 15 & Caucasian & Female & 38 & ADDwR & $x$ \\
\hline 16 & Caucasian & Female & 37 & ADDwR & $x$ \\
\hline 17 & Caucasian & Female & 36 & ADDwR & $x$ \\
\hline 18 & Caucasian & Female & 34 & ADDwR & \\
\hline 19 & Caucasian & Female & 46 & ADDwR & $x$ \\
\hline 20 & Caucasian & Female & 26 & ADDwR & $x$ \\
\hline 21 & Caucasian & Female & 30 & ADDwR & $x$ \\
\hline 22 & Caucasian & Female & 36 & ADDwR & $x$ \\
\hline 23 & Caucasian & Female & 44 & ADDwR & $x$ \\
\hline 24 & Caucasian & Female & 26 & ADDwR & $x$ \\
\hline 25 & Caucasian & Female & 17 & $\mathrm{CH}$ & \\
\hline 26 & Caucasian & Female & 43 & C FX & \\
\hline 27 & Caucasian & Female & 40 & $\mathrm{CH}$ & \\
\hline 28 & Caucasian & Female & 18 & $\mathrm{CH}$ & \\
\hline 29 & Caucasian & Male & 26 & C FX & $x$ \\
\hline 30 & Caucasian & Female & 30 & $\mathrm{CH}$ & \\
\hline 31 & $\begin{array}{l}\text { Afro- } \\
\text { American }\end{array}$ & Male & 26 & $\mathrm{CH}$ & $x$ \\
\hline 32 & Caucasian & Male & 27 & C FX & $x$ \\
\hline 33 & Caucasian & Male & 18 & C FX & \\
\hline
\end{tabular}


The subjects did not have any of the following: use of orthodontic appliances; chronic usage of anti-inflammatory drugs; history of diabetes, hepatitis, or HIV infection; immunosuppressive chemotherapy; history of any disease known to severely compromise immune function; current pregnancy or lactation; dentofacial deformity; major jaw trauma; previous TMJ surgery; and previous steroid injection in the TMJ. The subjects completed personal medical history questionnaires and, within a protocol approved by an Institutional Review Board, signed a consent form after being advised of the nature of the study. All patients were asked to complete a pain questionnaire, and an experienced oral and maxillofacial surgeon performed a clinical examination. The clinical examination consisted of palpating the TMJ region, noting the occurrence of painful opening/closing of the mouth, and listening for crepitation. The patients considered affected were treated surgically when they presented painful clinical signs of disk displacement after unsuccessful non-surgical treatment for at least 6 months. Patients presenting with pain related to muscular spasms were not included in this study. For the complementary exams, all patients received a panorex. These patients were from the Brazilian public health system; therefore, very few patients had the financial means to afford other exams, such as computerized tomography (CT) scan or a TMJ magnetic resonance imaging.

Accordingly, the diagnoses were primarily clinical. The subjects were included in clinical categories, according to the presence or absence of disk displacement.

1. Test group: composed of 36 samples, and all patients were diagnosed with one of the two following TMJ dysfunctions: (i) disk displacement with reduction (ADDwR, $n=29$ ) or (ii) disk displacement without reduction (ADDwoR, $n=7$ ). ADDwoR is considered the more severe condition.

2. Control group $(n=9)$ : composed of patients with no signs or symptoms of TMJ dysfunction, such as pain or mouth opening limitation. Of the control patients, four individuals presented condyle fractures (CFx) confirmed by radiographs and CT scans and needed surgery for fracture reduction, and five subjects displayed active condyle hyperplasia $(\mathrm{CH})$. Therefore, a wedge of the posterior region of the disks of all subjects was removed to physiologically adapt the disk on the condyle head.

Journal of Oral Pathology \& Medicine, Vol 44, No. 1 (January 2015): pg. 75-79. DOI. This article is (c) Wiley and permission has been granted for this version to appear in e-Publications@Marquette. Wiley does not grant permission for this article to be further copied/distributed or hosted elsewhere without the express permission from Wiley. 


\section{MMP-2 and MMP-9 immunohistochemical analysis}

The TMJ disk sections were deparaffinized with xylol

$(2 \times 10 \mathrm{~min})$ and rehydrated with absolute ethyl alcohol $(3 \times 1 \mathrm{~min})$ and $80 \%$ ethyl alcohol $(1 \times 1 \mathrm{~min})$.

Endogenous peroxidase activity was quenched by treatment with $\mathrm{H}_{2} \mathrm{O}_{2}$ ( $5 \%$ in methanol) for 10 min. Target Retrieval Solution ${ }^{\mathrm{TM}}$ (Dako, DK-2600 Glostrup, Denmark) was used prior to slide staining for heat-inducing epitope retrieval for formalin-fixed, paraffinembedded material, according to the manufacturer's instructions.

The sections were incubated with monoclonal MMP-2 and MMP-9 antibodies (Novocastra, New Castle, UK) diluted 1:50 in phosphatebuffered saline (PBS) and $0.1 \%$ bovine serum albumin (BSA). These monoclonal antibodies only stain a cellular fraction of MMP-2 and MMP9.

For negative controls, the primary antibody was not added, and PBS was used instead. The secondary antibody Advance ${ }^{\mathrm{TM}}$ (Dako, DK2600 Glostrup, Denmark) was applied for 30 min, according to the manufacturer's instructions.

The immunoreactions were visualized by incubating the sections with 1 drop 3,3' diaminobenzidine (DAB) chromogen (OriGene, Rockville, MD, USA) in $1 \mathrm{ml}$ distilled water. The sections were lightly counterstained with Harris hematoxylin for 5 min and finally mounted. The immunostaining was considered specific to MMP-2 and MMP-9 because immunoreactivity was not observed in the negative controls.

The positive areas were marked using the color morphometry method, which consisted of an analysis of the anti-MMP-2 and MMP-9 reaction area with the TMJ disk tissue. For this purpose, images of consecutive fields were captured with a $40 \times$ objective lens coupled to a BX50 Olympus microscope with a Sony camera (Model DXC-107A), and image analysis was performed with Image Pro Plus software (Media Cybernetics Inc., Silver Spring, MD, USA). This software allows an observer to select and paint the positive areas to obtain an image model and make a mask for staining the other slides. A single blinded observer performed this procedure. Moreover, the software 
automatically calculates the area of the positive reaction. The data were entered into a spreadsheet, and Microsoft Excel (Redmond, WA, USA) was used for the statistical analysis. The variable area was measured in square micrometers $\left(\mu \mathrm{m}^{2}\right)$ and was obtained with the mean of all positive areas.

\section{Statistical analysis}

SPSS version 14.0 (SPSS Institute, Inc., Chicago, IL, USA) was used for the statistical analyses. To analyze the area and density, the average of the results from all available samples of each patient was considered. Descriptive statistical values are presented as the mean and standard deviation, median, minimum, and maximum. Comparisons of the three groups were performed with one-way analysis of variance (ANOVA) and LSD (least significant difference) for multiple comparisons. To achieve the symmetric condition, the data were previously submitted to a logarithmic transformation. A value of $P<0.05$ indicates statistical significance.

\section{Results}

\section{MMP-2 expression analysis}

A significant difference was found between the groups with and without TMJ dysfunction $(P=0.030)$ for the MMP-2 area of expression (Table 2). There was increased MMP-2 immunostaining in the ADDwR group compared with the control group $(P=0.048)$. There was an increased level of expression in the ADDwoR group compared with the ADDwR group $(P=0.029$; Table $\underline{2})$. 
Table 2. Analysis of MMP-2 expression area between the groups

Variable Group $n$ Media Median Minimum Maximum Standard deviation

1. CG, control group; ADDwoR, disk displacement without reduction; ADDwR, disk displacement with reduction.

2. a One-way ANOVA, $P<0.05$ Area: $C G \times$ ADDwR: $P=0.048$; CG $\times$ ADDwoR: $P=0.725 ;$ ADDwR $\times$ ADDwoR: $P=0.029$.

$\begin{array}{lllllllll}\text { Area } & \text { CG } & 9 & 23.11 & 16.61 & 14.15 & 39.14 & 9.55 & 0.030 \\ \left(\mu \mathrm{m}^{2}\right) & \text { ADDwR } & 29 & 16.16 & 15.18 & 7.90 & 31.28 & 5.77 & \\ & \text { ADDwoR } 7 & 28.98 & 26.15 & 6.68 & 58.05 & 19.09 & \end{array}$

\section{MMP-9 expression analysis}

Comparison of the control and affected groups with or without reduction showed no significant difference for the MMP-9 area of immunostaining ( $P=0.476$; Table $\underline{3})$.

Table 3. Analysis of MMP-9 expression area between the groups

\section{Variable Group $n$ Media Median Minimum Maximum Standard P-valuea deviation}

1. CG, control group; ADDwoR, disk displacement without reduction; ADDwR, disk displacement with reduction.

2. a One-way ANOVA, $P<0.05$.

$\begin{array}{lllllllll}\text { Area } & \text { CG } & 9 & 21.2 & 15.8 & 6.8 & 53.9 & 15.7 & 0.476 \\ \left(\mu \mathrm{m}^{2}\right) & \text { ADDwR } & 31 & 18.1 & 16.1 & 2.1 & 102.6 & 17.6 & \\ & \text { ADDwoR } 7 & 29.5 & 21.8 & 6.8 & 101.3 & 32.8 & \end{array}$

\section{Discussion}

The articular disk of human in the TMJ adapts and remodels when subjected to loading. When the loading pressure reaches a high level, the inflammatory process is initiated and may entail the degradation and replacement of the natural matrix by abnormal tissue. 16 The upregulation of some MMPs has been observed in pathological conditions in the TMJ synovial fluid, resulting in tissue destruction, and MMPs are considered the primary enzymes involved in matrix turnover. $\frac{15,17-20}{1}$ MMP gene polymorphisms have been associated with temporomandibular joint degeneration, $\underline{\underline{21}}$ whereas MMP-9 gene 
promoter polymorphisms were associated with degenerative diseases, such as arthritis and atherosclerosis. $\underline{22}$

MMP-2 and MMP-9 expressions are regulated by several growth factors, such as platelet derived growth factor, tumor necrosis factor a, and interleukin-1. The latent presence of MMP-2 has been identified in healthy joints and is produced by fibroblasts, whereas MMP-9 is produced in large quantities by inflammatory cells, particularly in patients with osteoarthritis. $\underline{11}$ Both MMP-2 and MMP-9 are hypothesized to play multiple roles in TMJ pathogenesis because MMP-2 activates MMP-13 and because increased expression of MMP-2, concomitant with MMP-9, was observed in pathological conditions. $\frac{2,21}{}$ However, studies of these two enzymes have been performed in either animal models, cadavers, or the synovial fluid of human pathological TMJ. To the best of our knowledge, this is the first study to investigate the presence of MMP-2 and MMP-9 in human temporomandibular disks.

Our results showed statistically significant differences between CG and ADDwR and between ADDwoR and ADDwR. Notably, the expression of MMP-2 was also significantly increased in the disk displacement without reduction group compared with the disk displacement with reduction group and the control group. No difference was observed between the control group and ADDwoR. Although the lack of difference may be due to the small sample size and high variability in the expression of MMP-2 in these groups, it may also indicate the association of the increased expression of MMP-2 with a more severe TMJ disorder. The MMP-9 analysis showed no statistically significant difference between the groups in relation to the area of expression. The results of this study differ from the results of Yoshida et al. 11 who found higher levels of MMP-9 in the synovial fluid in advanced TMJ disease. Moreover, the lack of difference between the groups may be related to the small number of individuals and the high variability of MMP-9 expression within each group. Another possible explanation is that there may be a difference between MMP-9 expression in the synovial fluid and the disks during TMJ dysfunction. It is notable that although the sample size is reduced, only approximately $5 \%$ of people worldwide present a functional or pathological TMJ condition amenable to surgery, $\underline{23}$ and for ethical reasons, it is not possible to retrieve healthy retrodiscal tissue from normal patients to serve as controls. Moreover, the absence of MMP

Journal of Oral Pathology \& Medicine, Vol 44, No. 1 (January 2015): pg. 75-79. DOI. This article is @ Wiley and permission has been granted for this version to appear in e-Publications@Marquette. Wiley does not grant permission for this article to be further copied/distributed or hosted elsewhere without the express permission from Wiley. 
expression between the groups may be because the control group consisted of disks from patients with condyle fracture. However, for the controls presenting with mandibular condyle fracture, the surgical procedure occurred up to $24 \mathrm{~h}$ after the trauma, which diminishes the action of MMP degradation in the area. For the controls with hyperplasia, because hyperplasia is restricted to the condyle, this condition does not appear to affect the articular disk.

Although the sample size is small, to the best of our knowledge, this study has the largest sample of disks from living people, as opposed to disks from cadavers or from animals. Moreover, for human cadavers, no previous clinical records were available, which made it impossible to determine whether the subjects presented with any inflammatory degenerative alterations.

With an increasing number of patients presenting with TMJ dysfunction, the identification of the disease stage is a priority for directing proper treatment. In this study, increased expression of MMP-2 was observed in patients with disk displacement without reduction by immunohistochemistry analysis. No statistically significant difference was found in the area of expression of MMP-9, possibly because of the high interindividual variation in its expression, which therefore requires a larger number of individuals to determine whether the level of this protease is increased in the disks of individuals with TMJ dysfunction.

Future analyses of other MMPs combined with the results of this study may contribute to a better understanding of the role of MMPs on tissue remodeling and destruction. These studies may also improve the understanding of TMJ dysfunction and lead to improved patient treatments.

\section{References}

${ }^{1}$ Beek M, Aarnts MP, Koolstra JH, Feilzer AJ, van Eijden TM. Dynamic properties of the human temporomandibular joint disc. J Dent Res 2001; 80: 876-80.

${ }^{2}$ Marchetti C, Cornaglia I, Casasco A, Bernasconi G, Baciliero U, StetlerStevenson WG. Immunolocalization of gelatinase-A (matrix

Journal of Oral Pathology \& Medicine, Vol 44, No. 1 (January 2015): pg. 75-79. DOI. This article is (c) Wiley and permission has been granted for this version to appear in e-Publications@Marquette. Wiley does not grant permission for this article to be further copied/distributed or hosted elsewhere without the express permission from Wiley. 
metalloproteinase-2) in damaged human temporomandibular joint discs. Arch Oral Biol 1999; 44: 297-304.

${ }^{3}$ Westesson $\mathrm{P}-\mathrm{L}$, Bronstein SL, Liedberg J. Internal derangement of temporomandibular joint: morphologic description with correlation to function. Oral Surg Oral Med Oral Pathol 1985; 59: 323-31.

${ }^{4}$ Wilkes C. Arthrography of the temporomandibular joint in patients with the TMJ pain-dysfunction syndrome. Minutesn Med 1978; 61: 645-52.

${ }^{5}$ Makowski GS, Ramsby ML. Zymographic analysis of latent and activated forms of matrix metalloproteinase- 2 and 9 in synovial fluid: correlation to polymorphonuclear leukocyte infiltration and in response to infection. Clin Chim Acta 2003; 329: 77-81.

${ }^{6} \mathrm{Vu}$ TH, Werb Z. Matrix metalloproteinases: effectors of development and normal physiology. Genes Dev 2000; 14: 2123-33.

${ }^{7}$ Brew K, Dinakarpandian D, Nagase H. Tissue inhibitors of metalloproteinases: evolution, structure and function. Biochim Biophys Acta 2000; 1477: 267-83.

${ }^{8}$ Kozaci LD, Buttle DJ, Hollander AP. Degradation of type II collagen, but not proteoglycan, correlates with matrix metalloproteinase activity in cartilage explant cultures. Arthritis Rheum 1997; 40: 164-74.

${ }^{9}$ Nagase H, Woessner JF Jr. Matrix metalloproteinases. J Biol Chem 1999; 274: 21491-4.

${ }^{10}$ Makowski GS, Ramsby ML. Autoactivation profiles of calcium-dependent matrix metalloproteinase- 2 and -9 in inflammatory synovial fluid: effect of pyrophosphate and bisphosphonates. Clin Chim Acta 2005; 358: 182-91.

${ }^{11}$ Yoshida K, Takatsuka S, Hatada E, et al. Expression of matrix metalloproteinases and aggrecanase in the synovial fluids of patients with symptomatic temporomandibular disorders. Oral Surg Oral Med Oral Pathol Oral Radiol Endod 2006; 102: 22-7.

${ }^{12}$ Tanaka A, Kumagai S, Kawashiri S, et al. Expression of matrix metalloproteinase-2 and -9 in synovial fluid of the temporomandibular joint accompanied by anterior disc displacement. J Oral Pathol Med 2001; 30: 59-64.

${ }^{13}$ Mizui T, Ishimaru J, Miyamoto K, Kurita K. Matrix metalloproteinase-2 in synovial lavage fluid of patients with disorders of the temporomandibular joint. Br J Oral Maxillofac Surg 2001; 39: 310-4.

${ }^{14}$ Puzas JE, Landeau JM, Tallents R, Albright J, Schwarz EM, Landesberg R. Degradative pathways in tissues of the temporomandibular joint. Use of in vitro and in vivo models to characterize matrix metalloproteinase and cytokine activity. Cells Tissues Organs 2001; 169: 248-56.

${ }^{15}$ Leonardi R, Loreto $\mathrm{C}$, Barbato $\mathrm{E}$, et al. MMP-13 (collagenase 3) localization in human temporomandibular joint discs with internal derangement. Acta Histochem 2008; 110: 314-8.

Journal of Oral Pathology \& Medicine, Vol 44, No. 1 (January 2015): pg. 75-79. DOI. This article is (C) Wiley and permission has been granted for this version to appear in e-Publications@Marquette. Wiley does not grant permission for this article to be further copied/distributed or hosted elsewhere without the express permission from Wiley. 
NOT THE PUBLISHED VERSION; this is the author's final, peer-reviewed manuscript. The published version may be accessed by following the link in the citation at the bottom of the page.

${ }^{16}$ Buckwalter JA, Mankin HJ. Articular cartilage: tissue design and chondrocyte-matrix interactions. Instr Course Lect 1998; 47: 477-86.

${ }^{17}$ Kubota E, Kubota T, Matsumoto J, Shibata T, Murakami KI. Synovial fluid cytokines and proteinases as markers of temporomandibular joint disease. J Oral Maxillofac Surg 1998; 56: 192-8.

${ }^{18}$ Kubota $E$, Imamura $H$, Kubota $T$, Shibata $T$, Murakami K. Interleukin $1 \beta$ and stromelysin (MMP3) activity of synovial fluid as possible markers of osteoarthritis in the temporomandibular joint. J Oral Maxillofac Surg 1997; 55: 20-7.

${ }^{19}$ Ishimaru JI, Oguma Y, Goss AN. Matrix metalloproteinase and tissue inhibitor of metalloproteinase in serum and lavage fluid of patients with temporomandibular joint disorders. Br J Oral Maxillofac Surg 2000; 38: 354-9.

${ }^{20}$ Leonardi R, Almeida LE, Loreto $\mathrm{C}$. Lubricin immunohistochemical expression in human temporomandibular joint disc with internal derangement. $J$ Oral Pathol Med 2011; 40: 587-92.

${ }^{21}$ Planello AC, Campos MI, Meloto CB, et al. Association of matrix metalloproteinase gene polymorphism with temporomandibular joint degeneration. Eur J Oral Sci 2011; 119: 1-6.

${ }^{22}$ Clegg PD, Carter SD. Matrix metalloproteinase- 2 and -9 are activated in joint diseases. Equine Vet $\mathrm{J}$ 1999; 31: 324-30.

${ }^{23}$ Dimitroulis G. Temporomandibular joint surgery: what does it mean to the dental practitioner? Aust Dent J 2011; 56: 257-64.

Journal of Oral Pathology \& Medicine, Vol 44, No. 1 (January 2015): pg. 75-79. DOI. This article is (c) Wiley and permission has been granted for this version to appear in e-Publications@Marquette. Wiley does not grant permission for this article to be further copied/distributed or hosted elsewhere without the express permission from Wiley. 